\title{
ADSORÇÃo dE As, Cu, Pb E Cr NA AVALIAÇÃo dA CAPACIDADE DE FIXAÇÃo dE METAIS POR RESÍDUO DE MINERADORAS DE FERRO
}

\author{
Márcio Silva Basílio* \\ Centro Federal de Educação Tecnológica de Minas Gerais, Av. Amazonas 5253, 30480-000 Belo Horizonte - MG \\ Kurt Friese \\ Department Lake Research, Centre for Environmental Research, 39114 Magdeburg, Alemanha \\ Jorge Carvalho de Lena \\ Departamento de Química, Instituto de Ciências Exatas e Biológicas, Universidade Federal de Ouro Preto, \\ 35400-000 Ouro Preto - MG \\ Hermínio Arias Nalini Júnior e Hubert Mathias Peter Roeser \\ Departamento de Geologia, Escola de Minas, Universidade Federal de Ouro Preto, 35400-000 Ouro Preto - MG
}

Recebido em 9/8/04; aceito em 22/2/05; publicado na web em 30/6/05

\begin{abstract}
ADSORPTION OF As, $\mathrm{Cu}, \mathrm{Pb}$ AND Cr FOR THE ASSESSMENT OF METAL RETENTION BY TWO DIFFERENT RESIDUES FROM IRON ORE MINES. Two samples of residues from iron mining plants have been investigated for their retention capacity of $\mathrm{As}, \mathrm{Cu}, \mathrm{Cr}$, and $\mathrm{Pb}$. The sample with the higher content of iron oxides showed the highest capacity to retain metals. The adsorption affinity series changes from $\mathrm{Pb}>\mathrm{Cu}>\mathrm{Cr} \approx \mathrm{As}$ to $\mathrm{As}>\mathrm{Pb}>\mathrm{Cu}>\mathrm{Cr}$ or $\mathrm{As}>\mathrm{Cu}>\mathrm{Cr}>\mathrm{Pb}$, depending on the material and the concentration of the initial solution. In the competitive environment, the $\mathrm{Pb}$ adsorption decreases and the $\mathrm{As}, \mathrm{Cu}$ and $\mathrm{Cr}$ adsorption increases. Sequential extraction procedures, carried out after adsorption batch experiments, showed that the most important adsorption process occurs in the oxide fraction and that the major part of the absorbed metal is remobilized from exchangeable and oxide fractions.
\end{abstract}

Keywords: iron rich sediments; adsorption isotherm; sequential extraction.

\section{INTRODUÇÃO}

A adsorção de metais por superfícies minerais em geral e por óxidos de ferro em particular é, reconhecidamente, um importante processo que controla a geodisponibilidade, a mobilidade e, por consequiência, o destino e a biodisponibilidade dos metais em ambientes aquáticos ${ }^{1-4}$. Para se avaliar a disponibilidade dos metais é necessário entender sob quais condições eles estão associados aos sedimentos. $\mathrm{O}$ fracionamento por extração seqüencial e a construção de isotermas de adsorção fornecem valiosas informações acerca das relações entre metais e sedimentos.

$\mathrm{O}$ fracionamento por extração seqüencial permite identificar a distribuição dos metais nas várias fases sólidas de solos ou sedimentos $^{5-9}$. A evolução destes processos tem conduzido a tentativas de harmonização dos vários métodos de extração existentes. O estágio mais recente deste processo foi a certificação, pela Comissão Européia, de um método de extração seqüencial e de um material de referência para as extrações ${ }^{10-14}$.

A interpretação das isotermas de adsorção permite avaliar a capacidade de adsorção e a força com a qual os metais se ligam a determinado adsorvente ${ }^{15,16}$. Os mecanismos de adsorção podem ser descritos como resultado da formação de complexos entre os grupos funcionais (hidroxilas) expostos na superfície mineral e as espécies químicas presentes em solução ${ }^{17}$. As isotermas podem ser construídas empiricamente ou obtidas através de modelagem matemática (modelos de complexação superficial). Estes modelos procuram descrever os processos de adsorção baseados na associação entre a equação da lei de massas e os efeitos eletrostáticos causados pelas variações nas cargas das superfícies minerais ${ }^{18-20}$. Os modelos de complexação superficial explicam satisfatoriamen-

*e-mail: marcio.basilio@uol.com.br te o fenômeno de adsorção nos sistemas simples, onde apenas uma fase mineral encontra-se presente, mas existem dúvidas acerca da manutenção das propriedades adsortivas dos minerais quando presentes em misturas, como no caso dos materiais naturais, como solos, sedimentos e resíduos industriais ${ }^{17}$.

Neste trabalho, o material estudado provém do Quadrilátero Ferrífero, Minas Gerais. Esta região, considerada uma das mais mineralizadas do globo, vem sendo intensamente minerada desde meados do século XVII. Metais associados aos minérios foram sendo historicamente liberados para os cursos d'água que drenam a região, provocando contaminação da água e dos sedimentos ${ }^{21-23}$.

O material estudado foi coletado em duas barragens que funcionam como primeira (Timbopeba) e segunda (Natividade) bacias de decantação, onde são depositadas misturas de rejeitos, material erodido nas sub-bacias dos córregos Timbopeba e Natividade e lama residual do processamento primário do minério de ferro proveniente da planta de tratamento de Timbopeba de propriedade da Cia. Vale do Rio Doce. Considerando sua forma de ocorrência, este material será chamado doravante de sedimento. A principal diferença entre os sedimentos coletados nas Barragens de Timbopeba e Natividade consiste no teor de óxidos de ferro ${ }^{24}$.

Considerando que a matriz dos sedimentos é composta basicamente por uma mistura de óxidos de ferro e aluminossilicatos, minerais cada vez mais utilizados como imobilizadores de metais em áreas contaminadas ${ }^{25-31}$, e o baixo custo do processo, este trabalho objetivou avaliar o uso dos sedimentos das barragens de contenção do processamento do minério de ferro como minimizador da contaminação de metais em águas por processos de adsorção nas fases minerais do sedimento.

Para atingir este objetivo e considerando a complexidade dos processos de retenção de metais por sedimentos, este trabalho foi desenvolvido em três partes com a utilização de diferentes méto- 
dos de maneira associada ${ }^{32-35}:$ (1) fracionamento por extração seqüencial dos sedimentos, (2) construção de isotermas de adsorção e (3) adsorção de metais seguida de extração dos sedimentos. A parte 1 buscou identificar a distribuição de $\mathrm{As}, \mathrm{Cu}, \mathrm{Cr}$ e $\mathrm{Pb}$ nas várias fases dos sedimentos, através de procedimentos de fracionamento por extração seqüencial, permitindo avaliar a geodisponibilidade dos metais originalmente presentes nestes sedimentos.

A parte 2 objetivou quantificar a capacidade de adsorção das superfícies para $\mathrm{As}, \mathrm{Cu}, \mathrm{Cr}$ e $\mathrm{Pb}$ em condições não competitivas, pela construção de isotermas com apenas um metal em solução e em condições competitivas, a partir de soluções onde os 4 elementos estavam presentes.

A parte 3 foi executada com o objetivo de identificar e quantificar a capacidade de adsorção de $\mathrm{As}, \mathrm{Cu}, \mathrm{Cr}$ e $\mathrm{Pb}$ por cada fase presente nos sedimentos, tanto em condições competitivas quanto não competitivas. A partir destes dados pôde-se avaliar o tipo de associação que estes metais fazem com os sedimentos e, conseqüentemente, complementar as informações sobre a geodisponibilidade em relação aos metais que porventura venham a ser adsorvidos por estes sedimentos.

\section{PARTE EXPERIMENTAL}

\section{Vidraria e reagentes}

Todas as vidrarias e frascos utilizados nos procedimentos descritos neste trabalho foram imersos em solução de $\mathrm{HNO}_{3} 4 \mathrm{~mol} \mathrm{~L}^{-1}$ por $24 \mathrm{~h}$ e enxaguados abundantemente em água destilada-deionizada. Todos os reagentes utilizados eram de alta pureza e as soluções foram preparadas utilizando-se água superpura (Milli-Q, Millipore ${ }^{\circledR}$ ).

\section{Sedimentos}

Os sedimentos foram coletados em dois pontos, um em cada reservatório, recuperando-se, com auxílio de um amostrador tipo Ekman, cerca de $5 \mathrm{~kg}$ de sedimento por ponto amostrado. O material foi seco à temperatura ambiente, cuidadosamente descompactado utilizando-se gral de ágata, quarteado e acondicionado em frascos de polipropileno. Em subamostras destes sedimentos foi caracterizado o conteúdo de nitrogênio, enxofre, carbono orgânico e inorgânico (Elementar-Analysator-Vario EL, Elementar Analysensystem), distribuição granulométrica (Particle sizer PARTmaster L, Aucoteam GmbH), capacidade de troca catiônica (CTC - saturação com $\mathrm{NH}_{4} \mathrm{Cl}$ ), mineralogia (difratômetro de Raios $\mathrm{X}$ - Bruker D5000, Siemens) e área superficial (BET-N 2 - Gemini 2360 - V5.00, Micromeritics).

\section{Fracionamento}

Adotou-se o método de fracionamento por extração seqüencial em 3 etapas estabelecida pelo "Standard, Measurements and Testing Program - BCR" ${ }^{36,37}$. As extrações foram feitas em triplicatas, usando-se alíquotas de 1.000,0 $( \pm 0,2)$ mg de sedimento seco. Os resultados foram controlados em relação aos valores certificados (etapas 1 a 3) e valores indicativos (digestão pseudototal do resíduo da etapa 3 e do material integral) para $\mathrm{Cu}, \mathrm{Cr}$ e $\mathrm{Pb}$ com a utilização do material de referência BCR-701. Após cada etapa de extração, as suspensões foram centrifugadas por 20 min à velocidade de 3.000 $\mathrm{x} g$, os extratos transferidos para frascos de polipropileno e armazenados a $4{ }^{\circ} \mathrm{C}$ até o momento da análise. Todas as etapas, incluindo a residual, foram executadas em um mesmo tubo de policarbonato a fim de minimizar a perda ou a contaminação do material. Entre cada etapa, os resíduos foram lavados com $20 \mathrm{~mL}$ de água. Os extratos foram analisados por espectrometria de massas com fonte de plasma (ICP-MS, Agilent 7500c).

Etapa 1 - Íons trocáveis e íons associados a carbonatos: $40 \mathrm{~mL}$ de ácido acético $0,11 \mathrm{~mol} \mathrm{~L}^{-1}$ foram adicionados aos tubos contendo a alíquota do sedimento. As suspensões foram agitadas em agitador rotativo $360^{\circ}$ (Heidolph Instruments, Reax 2) por $16 \mathrm{~h}$.

Etapa 2 - Fase redutível ou íons associados a óxidos de ferro e de manganês: $40 \mathrm{~mL}$ de cloridrato de hidroxilamina $0,1 \mathrm{~mol} \mathrm{~L}^{-1}$ ( $\mathrm{pH} 2$ com $\mathrm{HNO}_{3}$ 15,8 mol L-1) foram adicionados aos tubos com os resíduos da Etapa 1. As suspensões foram agitadas por $16 \mathrm{~h}$.

Etapa 3 - Fase oxidável ou metais associados a sulfetos e matéria orgânica: $10 \mathrm{~mL}$ de peróxido de hidrogênio $8,8 \mathrm{~mol} \mathrm{~L}^{-1}$ foram adicionados aos tubos contendo os resíduos da etapa anterior. A extração ocorreu a frio por $1 \mathrm{~h}$ e a $85 \pm 5^{\circ} \mathrm{C}$, com eventual agitação manual, até redução do volume para $3 \mathrm{~mL}$. Mais uma alíquota de $10 \mathrm{~mL}$ de peróxido de hidrogênio $8,8 \mathrm{~mol} \mathrm{~L}^{-1}$ foi adicionada aos tubos e as suspensões foram reaquecidas a $85 \pm 5^{\circ} \mathrm{C}$ até próximo à secura. Após resfriamento foram adicionados em cada tubo $50 \mathrm{~mL}$ de acetato de amônio 1,0 mol L-1 (pH 2 com $\mathrm{HNO}_{3}$ 15,8 mol L-1) para prevenir readsorção. As suspensões foram agitadas por $16 \mathrm{~h}$.

Ao resíduo da Etapa 3 e às alíquotas do sedimento integral $(1.000,0 \pm 0,2 \mathrm{mg})$, foram adicionados $9,3 \mathrm{~mL}$ de água régia $(7,0$ $\mathrm{mL}$ de $\mathrm{HCl} 12,0 \mathrm{~mol} \mathrm{~L}^{-1}$ e 2,3 mL de $\left.\mathrm{HNO}_{3} 15,8 \mathrm{~mol} \mathrm{~L}^{-1}\right)$. A digestão ocorreu a frio por $16 \mathrm{~h}$ e, em seguida, as suspensões foram aquecidas por $2 \mathrm{~h}$ a $90 \pm 5^{\circ} \mathrm{C}$ com os tubos parcialmente tampados. O material foi então filtrado e o volume completado para 50 $\mathrm{mL}$.

A digestão com água régia, tanto do resíduo da fase 3 da extração quanto do sedimento integral, é recomendada pelo método BCR. Este procedimento, referido como "digestão pseudototal", disponibiliza os metais associados às fases minerais residuais (óxidos fortemente cristalinos e alguns silicatos ${ }^{38,39}$ ) sendo considerado um controle de qualidade vital para o protocolo BCR 9 .

\section{Isotermas de adsorção}

Foram construídas isotermas de adsorção competitivas e não competitivas para $\mathrm{As}, \mathrm{Cu}, \mathrm{Cr}$ e $\mathrm{Pb}$ nos dois sedimentos estudados, utilizando-se a metodologia proposta por Echeverría et al ${ }^{33}$. Cada isoterma não competitiva foi desenvolvida utilizando-se, para cada sedimento, uma bateria de 16 soluções contendo um único elemento em concentrações crescentes. As isotermas competitivas foram construídas utilizando-se 16 soluções com misturas equimolares dos quatro elementos. As concentrações iniciais destas 16 soluções variaram entre 0,01 a $1,0 \mathrm{mmol} \mathrm{L}^{-1}$ e foram preparadas por diluição para $1 \mathrm{~L}$ de padrões Titrisol ${ }^{\circledR} 1.000 \mathrm{mg}$ (Merck, Darmstadt, Alemanha - $\mathrm{Cu}$ e $\mathrm{Cr}$ - cloreto, $\mathrm{Pb}$ - nitrato e As - ácido arsênico). Foram adicionados $25 \mathrm{~mL}$ de cada solução a tubos de polipropileno contendo $100,0 \pm 0,2 \mathrm{mg}$ de sedimento seco. As suspensões foram agitadas por $24 \mathrm{~h}$ em agitador rotativo em temperatura ambiente controlada $\left(23 \pm 1{ }^{\circ} \mathrm{C}\right)$ e centrifugadas por $20 \mathrm{~min}$ a $3.000 \mathrm{x} \mathrm{g}$. As soluções iniciais possuíam pH entre 5 e 4 e estes valores permaneceram praticamente estáveis até o fim do processo. As soluções centrifugadas foram estabilizadas com $100 \mathrm{~mL}$ de $\mathrm{HNO}_{3}\left(15,8 \mathrm{~mol} \mathrm{~L}^{-1}\right)$ e armazenadas em tubos de polipropileno a 4 ${ }^{\circ} \mathrm{C}$ para posterior análise via ICP-MS. As isotermas foram representadas graficamente relacionando-se a quantidade do metal adsorvido $(C s)$ em mmol kg-1 em função da concentração do metal que permaneceu em equilíbrio $(\mathrm{Ce})$ em $\mathrm{mmol} \mathrm{L}^{-1}$. O valor de $C e$ foi obtido diretamente a partir da análise da solução ao fim do procedimento e o valor de $C s$ obtido por diferença entre a concentração 
Tabela 1. Concentrações iniciais de $\mathrm{Cu}, \mathrm{Cr}$, As e Pb utilizadas no procedimento de adsorção para os sedimentos T-23 e N-31. Os valores indicam a concentração suficiente de cada elemento para saturar a superfície dos sedimentos $\left(C o_{a d s} \mathrm{em} \mathrm{mol} \mathrm{L}^{-1}\right)$

\begin{tabular}{|c|c|c|c|c|c|c|c|c|}
\hline & \multicolumn{4}{|c|}{$T-23$} & \multicolumn{4}{|c|}{$N-31$} \\
\hline branco & 0 & 0 & 0 & 0 & 0 & 0 & 0 & 0 \\
\hline \multirow{4}{*}{ não competitivo } & 2,4 & - & - & - & 2,7 & - & - & - \\
\hline & - & 3,3 & - & - & - & 2,5 & - & - \\
\hline & - & - & 2,0 & - & - & - & 2,1 & - \\
\hline & - & - & - & 0,8 & - & - & - & 0,7 \\
\hline competitivo & 2,4 & 3,3 & 2,0 & 0,8 & 2,7 & 2,5 & 2,1 & 0,7 \\
\hline
\end{tabular}

inicial $(C o)$ em mmol L ${ }^{-1}$ e a concentração no equilíbrio, pela utilização da fórmula:

$C s=\frac{(C o-C e) V}{W}$

onde $V$ é o volume da solução em L e $W$, o peso em kg do sedimento seco.

\section{Adsorção e extração dos sedimentos}

A adsorção seguida de extração foi realizada para os dois sedimentos em relação a $\mathrm{As}, \mathrm{Cu}, \mathrm{Cr}$ e $\mathrm{Pb}$ em dois níveis distintos: não competitivo e competitivo (Tabela 1). A concentração inicial das soluções usadas no procedimento de adsorção $\left(\mathrm{Co}_{a d s}\right)$ foi determinada a partir das isotermas não competitivas de modo a fornecer a quantidade mínima de íons suficiente para saturar as superfícies destes sedimentos. Foram determinados os valores de $\mathrm{Cs}$ e $\mathrm{Ce}$ referentes ao início do patamar de cada isoterma como exemplificado na Figura 1 (Cr no sedimento N-31). Para as isotermas que não se estabilizaram dentro do intervalo de concentrações estudado, foram usados os valores referentes ao término de sua parte mais íngreme.

As amostras de sedimento saturadas com metais foram preparadas, para cada elemento, pela adição de $25 \mathrm{~mL}$ de solução contendo a massa do íon necessária para a saturação do sedimento (Csm) somada à massa do íon que deverá sobrar em equilíbrio na solução $(\mathrm{Ce})$. As concentrações das soluções usadas são apresenta-

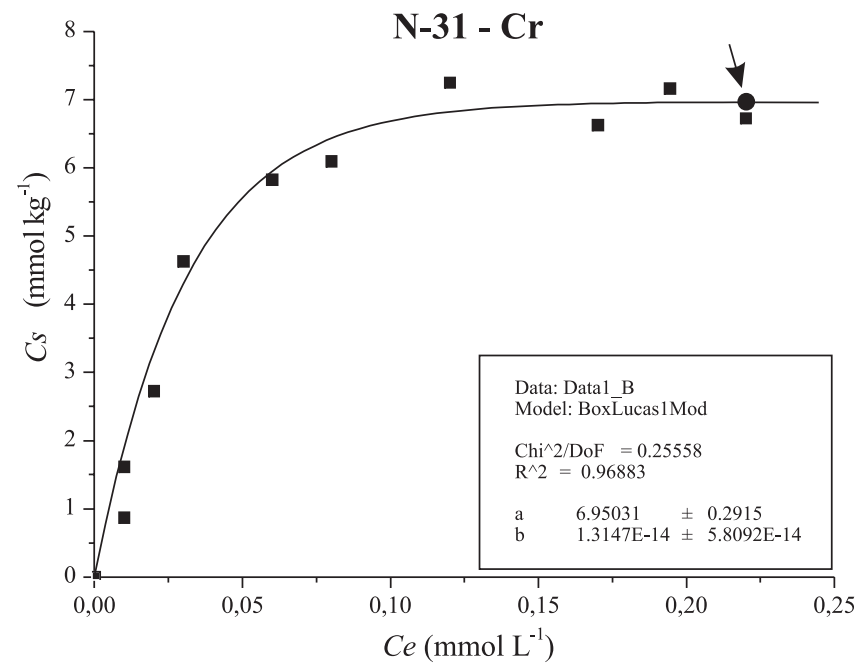

Figura 1. Isoterma de Cr no sedimento $N$-31. O parâmetro "a" indica o valor de Csm e a seta aponta a localização do par (Csm, Ce) usado para o cálculo de Co ${ }_{\text {ads }}$ das na Tabela 1. O experimento foi executado adicionando-se $25 \mathrm{~mL}$ da solução contendo o elemento estudado, ou a respectiva mistura no caso de adsorção competitiva, a 1.000,0 $\pm 0,2 \mathrm{mg}$ do sedimento. Cada suspensão foi agitada por $16 \mathrm{~h}$ em temperatura ambiente controlada $\left(23 \pm 1^{\circ} \mathrm{C}\right)$ e centrifugada por $20 \mathrm{~min}$ a 3.000 $\mathrm{x} g$. A solução foi desprezada e os sedimentos lavados duas vezes com $20 \mathrm{~mL}$ de água destilada-deionizada, centrifugados e extraídos pelo método BCR, de acordo com a metodologia já descrita.

\section{RESULTADOS E DISCUSSÃO}

\section{Sedimentos}

A principal diferença entre os sedimentos analisados consiste no seu teor de ferro, 46,5\% em T-23 e 11,2\% em N-31. Conseqüentemente, o sedimento N-31 apresenta maior teor de Al. A Tabela 2 caracteriza cada sedimento em relação a suas principais propriedades físicas e químicas.

Tabela 2. Resultados das análises de caracterização dos sedimentos T-23 e N-31

\begin{tabular}{lcc}
\hline & T-23 & $\mathbf{N}-31$ \\
\hline granulometria (\%) & & \\
$\quad>20 \mu \mathrm{m}$ & 1,0 & 4,3 \\
$\quad 20-2 \mu \mathrm{m}$ & 86,6 & 87,7 \\
$\quad<2 \mu \mathrm{m}$ & 12,4 & 8,0 \\
CTC (cmol(+) $\left.\mathrm{kg}^{-1}\right)$ & 0,92 & 0,55 \\
\% em base (\%) & 100 & 97 \\
área superficial BET-N ${ }_{2}$ & 9,7 & 8,7 \\
$\left(\mathrm{~m}^{2} \mathrm{~g}^{-1}\right)$ & & \\
mineralogia* & hem, goe, & hem, goe, \\
& mus, clo, cau, & mus, clo, \\
& alb, qtz & $\mathrm{qtz}$ \\
teor de Fe (\%) & 46,5 & 11,2 \\
teor de Al (\%) & 1,8 & 4,0 \\
pH (lama "in situ") & 7,3 & 7,2 \\
carbono total $\left(\mathrm{g} \mathrm{kg}^{-1}\right)$ & 2,8 & 2,6 \\
carbono inorgânico $\left(\mathrm{g} \mathrm{kg}^{-1}\right)$ & 0,1 & 0,2 \\
carbono orgânico $\left(\mathrm{g} \mathrm{kg}^{-1}\right)$ & 2,7 & 2,4 \\
nitrogênio total $\left(\mathrm{g} \mathrm{kg}^{-1}\right)$ & 0,4 & 0,4 \\
enxofre (g kg-1) & 0,8 & 0,8
\end{tabular}

* hem - hematita; qtz - quartzo; goe - goethita; cau - caulinita; mus - muscovita; alb - albita; clo - clorita

\section{Fracionamento}

O controle das extrações, feito através da extração do material de referência BCR-701 em paralelo aos sedimentos, aponta que a grande maioria dos valores obtidos encontra-se dentro do intervalo 
certificado (Tabela 3). O balanço de massa das extrações seqüenciais foi obtido somando-se os valores encontrados em cada etapa de extração dos sedimentos e comparando-se aos valores obtidos na digestão pseudototal do sedimento integral (Tabela 4). Este balanço indica que a extração seqüiencial foi eficiente para $\mathrm{Cu}, \mathrm{As}$ e $\mathrm{Cr}$. $\mathrm{A}$ extração do $\mathrm{Pb}$ foi comprometida nos dois sedimentos. A causa provável para esta pequena recuperação pode ser devida aos baixos teores de chumbo que, na maioria das frações, estão próximos ao limite de detecção do método analítico utilizado. Baixas concentrações de metais podem favorecer a contaminação ${ }^{40}$ e evidenciar o processo de readsorção ${ }^{41}$, inerentes aos procedimentos de extração seqüencial.

Os resultados do fracionamento e da digestão parcial dos sedimentos T-23 e N-31 integrais (Tabela 4) mostram que, exceto para $\mathrm{Cu}$, o sedimento proveniente da Barragem de Natividade apresenta maior concentração de metais que o da Barragem de Timbopeba. A distribuição dos elementos mostra que As e Cr ocorrem associados quase que exclusivamente à fração residual em ambos os sedimentos. $\mathrm{O} \mathrm{Cu}$ ocorre distribuído entre a fração residual, a fração óxido e a fração íons trocáveis. $\mathrm{O} \mathrm{Pb}$ aparece associado principalmente à fração óxido, seguido da fração sulfeto-matéria orgânica. Na fração íons trocáveis e na fração sulfeto-matéria orgânica os quatro elementos estudados estão presentes em concentrações muito baixas, em alguns casos abaixo do limite de detecção do método analítico utilizado.

\section{Isotermas de adsorção}

Isotermas não competitivas

As isotermas não competitivas de $\mathrm{As}, \mathrm{Cu}, \mathrm{Cr}$ e $\mathrm{Pb}$ para os sedimentos T-23 e N-31 estão representadas na Figura 2. Para facilitar a comparação, as isotermas, mesmo tendo sido construídas em experimentos independentes, foram agrupadas em um único gráfico por sedimento. Estas isotermas foram classificadas como Tipo I na classificação de Brunauer ${ }^{42}$ que se enquadra no modelo de isoterma de Langmuir. A comparação entre as quantidades adsorvidas de cada
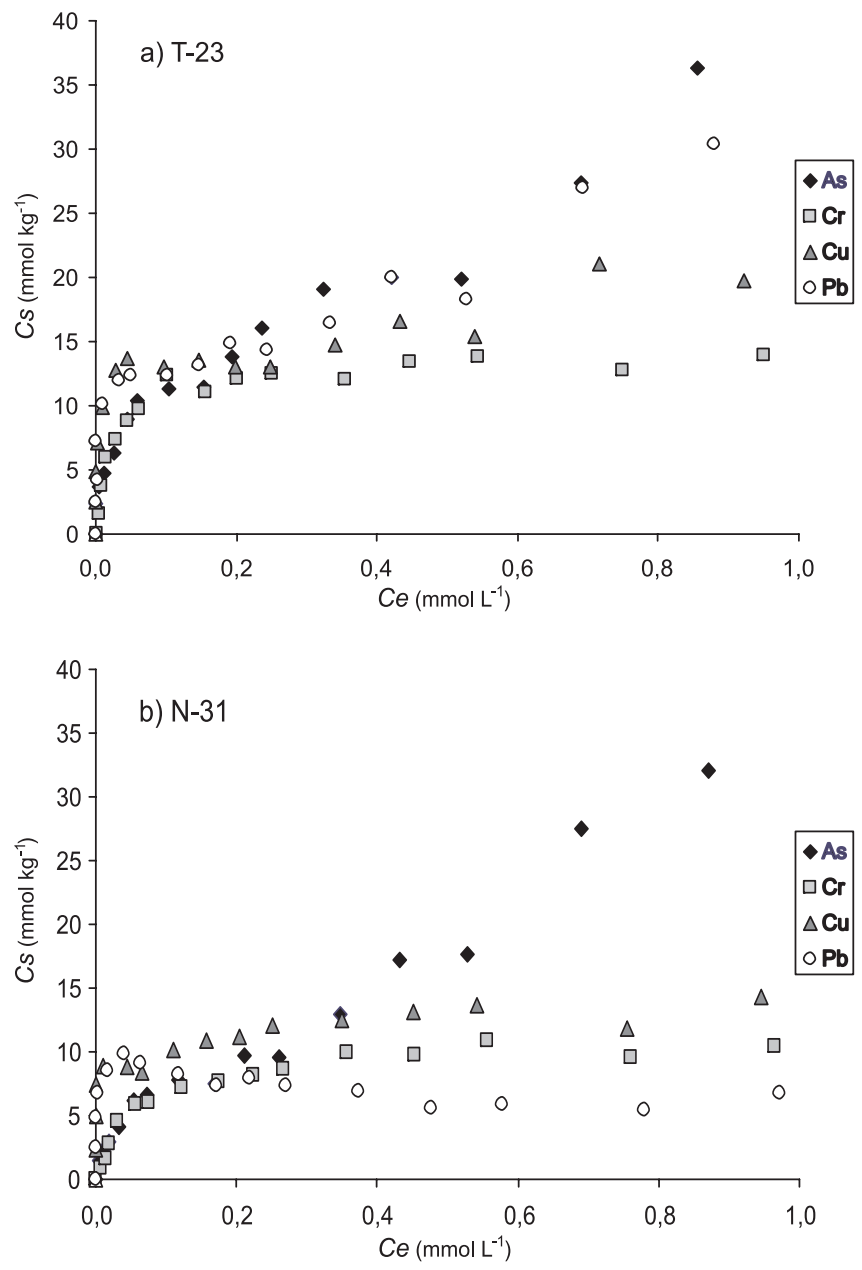

Figura 2. Isotermas não competitivas para $\mathrm{As}, \mathrm{Cu}, \mathrm{Cr}$ e $\mathrm{Pb}$ nos sedimentos $\mathrm{T}$ 23 e $N-31$

Tabela 3. Resultado das três etapas de extração do material de referência BCR-701 e das digestões pseudototais do resíduo da etapa 3 e do material de referência integral

\begin{tabular}{lccccccccccccccccc}
\hline & \multicolumn{3}{c}{ Etapa 1 } & \multicolumn{4}{c}{ Etapa 2 } & \multicolumn{3}{c}{ Etapa 3 } & \multicolumn{4}{c}{ Resíduo } & \multicolumn{4}{c}{ Pseudototal } \\
& VO & CV & VC & VO & CV & VC & VO & CV & VC & VO & CV & VI & VO & CV & VI \\
\hline $\mathrm{Cu}$ & 50,1 & $(1,5)$ & $49,3 \pm 1,7$ & 116 & $(6,0)$ & $124 \pm 3$ & 53,6 & $(0,4)$ & $55,2 \pm 4$ & 38,0 & $(0,9)$ & $38,5 \pm 11,2$ & 273 & $(1,2)$ & $275 \pm 13$ \\
$\mathrm{Cr}$ & 2,39 & $(3,0)$ & $2,26 \pm 0,16$ & 45,1 & $(0,6)$ & $45,7 \pm 2$ & 130 & $(2,0)$ & $143 \pm 7$ & 57,6 & $(1,6)$ & $62,5 \pm 7,4$ & 254 & $(2,9)$ & $272 \pm 20$ \\
$\mathrm{~Pb}$ & 3,15 & $(3,6)$ & $3,18 \pm 0,21$ & 126 & $(0,5)$ & $126 \pm 3$ & 7,8 & $(6,6)$ & $9,3 \pm 2$ & 23,0 & $(3,8)$ & $11 \pm 5,2$ & 144 & $(3,1)$ & $143 \pm 6$ \\
\hline
\end{tabular}

Valor obtido na análise (VO), valores certificados para o material de referência e suas incertezas (VC) e valor indicativo para o material de referência (VI) em $\mathrm{mg} \mathrm{kg}^{-1}$. Coeficiente da variação $(\mathrm{CV}, \mathrm{n}=3)$ em porcentagem.

Tabela 4. Distribuição de $\mathrm{Cu}, \mathrm{Cr}$, As e $\mathrm{Pb}$, em mg kg-1, nas frações dos sedimentos T-23 e N-31, obtida por extração seqüencial e digestão pseudototal do resíduo da extração e do sedimento integral

\begin{tabular}{|c|c|c|c|c|c|c|c|}
\hline & & Etapa 1 & Etapa 2 & Etapa 3 & Resíduo & Soma & Sed. integral \\
\hline \multirow[t]{4}{*}{$\mathrm{T}-23$} & $\mathrm{Cu}$ & $1,9(2,2)$ & $4,2(0,1)$ & $0,2 \quad(8,7)$ & $26,0 \quad(8,0)$ & 32,3 & $32,0 \quad(1,3)$ \\
\hline & $\mathrm{Cr}$ & $<0,02$ & $0,1 \quad(6,2)$ & $0,2 \quad(5,0)$ & $28,0 \quad(4,6)$ & 28,3 & $28,4 \quad(2,4)$ \\
\hline & As & $<0.02$ & $<0,02$ & $<0,02$ & $19,3 \quad(12,1)$ & 19,3 & $19,8 \quad(3,1)$ \\
\hline & $\mathrm{Pb}$ & $<0,02$ & $1,9(0,6)$ & $0,8 \quad(10,4)$ & $<0,02$ & 2,7 & $9,5 \quad(4,3)$ \\
\hline \multirow[t]{4}{*}{$\mathrm{N}-31$} & $\mathrm{Cu}$ & $1,4(8,1)$ & $3,1 \quad(2,2)$ & $0,8 \quad(9,7)$ & $4,8 \quad(7,1)$ & 10,1 & $13,6 \quad(4,8)$ \\
\hline & $\mathrm{Cr}$ & $<0,02$ & $<0,02$ & $<0,02$ & $102(6,9)$ & 102 & $91,5 \quad(5,2)$ \\
\hline & As & $<0,02$ & $1,2(1,0)$ & $<0,02$ & $62,3 \quad(7,1)$ & 61,4 & $62,3 \quad(4,0)$ \\
\hline & $\mathrm{Pb}$ & $<0,02$ & $13,5(1,0)$ & $1,5 \quad(8,7)$ & $<0,02$ & 15,1 & $22,2 \quad(8,7)$ \\
\hline
\end{tabular}

Os coeficientes de variação $(n=3)$, em porcentagem, são mostrados entre parêntesis. 
elemento nos dois sedimentos foi feita identificando-se a concentração de adsorção máxima $(C s m)$. Chumbo e $\mathrm{Cu}$ apresentam maior afinidade com os sedimentos, principalmente em baixas concentrações de equilíbrio $(\mathrm{Ce})$. A altura das isotermas não competitivas nos dois sedimentos indica que o material mais rico em ferro - T-23 apresenta maior capacidade de adsorção. Em ambos os sedimentos a afinidade para adsorção dos elementos a baixas concentrações de equilíbrio $(\mathrm{Ce})$ é $\mathrm{Pb}>\mathrm{Cu}>\mathrm{Cr} \approx$ As. Esta seqüência de afinidade de adsorção está de acordo com os resultados encontrados por vários pesquisadores ${ }^{1-3,33}$. A diferença entre as inclinações iniciais e a diferença entre alturas dos patamares de cada isoterma fazem com que esta relação de afinidades se modifique à medida que os valores de $\mathrm{Ce}$ crescem. No sedimento T-23 para $\mathrm{Ce}>0,2 \mathrm{mmol} \mathrm{L}^{-1}$ a afinidade de adsorção passa a ser $\mathrm{As}>\mathrm{Pb}>\mathrm{Cu}>\mathrm{Cr}$ (Figura 2a). No sedimento N-31 para $C e>0,3 \mathrm{mmol} \mathrm{L}^{-1}$ a afinidade de adsorção se altera para As $>\mathrm{Cu}>\mathrm{Cr}>\mathrm{Pb}$ (Figura 2b).

Quanto à forma, as isotermas não competitivas de As apresentam-se bastante semelhantes nos dois sedimentos. A ausência de um patamar nas isotermas indica que, dentro do intervalo de concentrações estudado, o equilíbrio de adsorção de As não foi atingido. A maior afinidade do As pelo sedimento T-23 pode ser observada comparando-se as isotermas nas Figuras $2 \mathrm{a}$ e $2 \mathrm{~b}$.

As isotermas de $\mathrm{Cu}$ apresentam comportamento semelhante nos dois sedimentos, mas indicam maior afinidade do $\mathrm{Cu}$ pelo sedimento T-23. Neste sedimento ocorre uma rápida adsorção de $\mathrm{Cu}$, representada na isoterma por uma forte inclinação inicial. No sedimento N-31, apesar da inclinação inicial da isoterma ser semelhante à encontrada em T-23, a saturação da superfície ocorre em um valor de $C s$ mais baixo.

A forma das isotermas de $\mathrm{Cr}$ assemelha-se bastante nos dois sedimentos. Entretanto, a diferença nas alturas dos patamares indica afinidade do $\mathrm{Cr}$ um pouco maior para com o sedimento T-23.

As isotermas do $\mathrm{Pb}$ mostram comportamento diferente entre os dois sedimentos. Em T-23 a isoterma do $\mathrm{Pb}$ não se estabiliza dentro do intervalo de concentrações estudado, mostrando, ao final deste intervalo, adsorção na ordem de $30 \mathrm{mmol} \mathrm{kg}{ }^{-1}$. Já no sedimento N-31, a isoterma estabiliza-se rapidamente $(\mathrm{Ce} \approx 0,1 \mathrm{mmol}$ $\mathrm{L}^{-1}$ ) com a adsorção de cerca de $8 \mathrm{mmol} \mathrm{L}^{-1}$.

\section{Isotermas competitivas}

As isotermas de adsorção competitivas, quando comparadas às isotermas não competitivas, indicam uma diminuição na adsorção de $\mathrm{Pb}$ e um aumento na adsorção de $\mathrm{Cr}, \mathrm{Cu}$ e As (Figura 3).

Em forma, as isotermas competitivas de As assemelham-se muito às respectivas isotermas não competitivas. Entretanto, a maior inclinação inicial e os valores mais elevados de $C$ s refletem o aumento da capacidade de adsorção de As em condições de competição. Como a espécie de As presente é o ânion arsenato, este aumento da adsorção ocorre, provavelmente, como resultado da combinação de efeitos eletrostáticos e possível formação de complexos ternários tipo superfície-metal-arsenato ${ }^{3,43}$ :

$\equiv \mathrm{FeOH}+\mathrm{M}^{+z}+\mathrm{L}^{-} \leftrightarrows \mathrm{FeLM}^{+}+\mathrm{OH}^{-}$

As isotermas competitivas de $\mathrm{Cr}$ e $\mathrm{Cu}$ apresentam comportamento semelhante. São íngremes e não se estabilizam dentro do intervalo estudado nos dois sedimentos. A comparação com as isotermas não competitivas mostra que o ambiente competitivo favorece a adsorção destes metais em ambos os sedimentos.

As isotermas competitivas de $\mathrm{Pb}$ são semelhantes nos dois sedimentos, mostrando uma diminuição da adsorção em condições competitivas. Este comportamento do $\mathrm{Pb}$ concorda com os resultados descritos em outros trabalhos ${ }^{34,44}$.
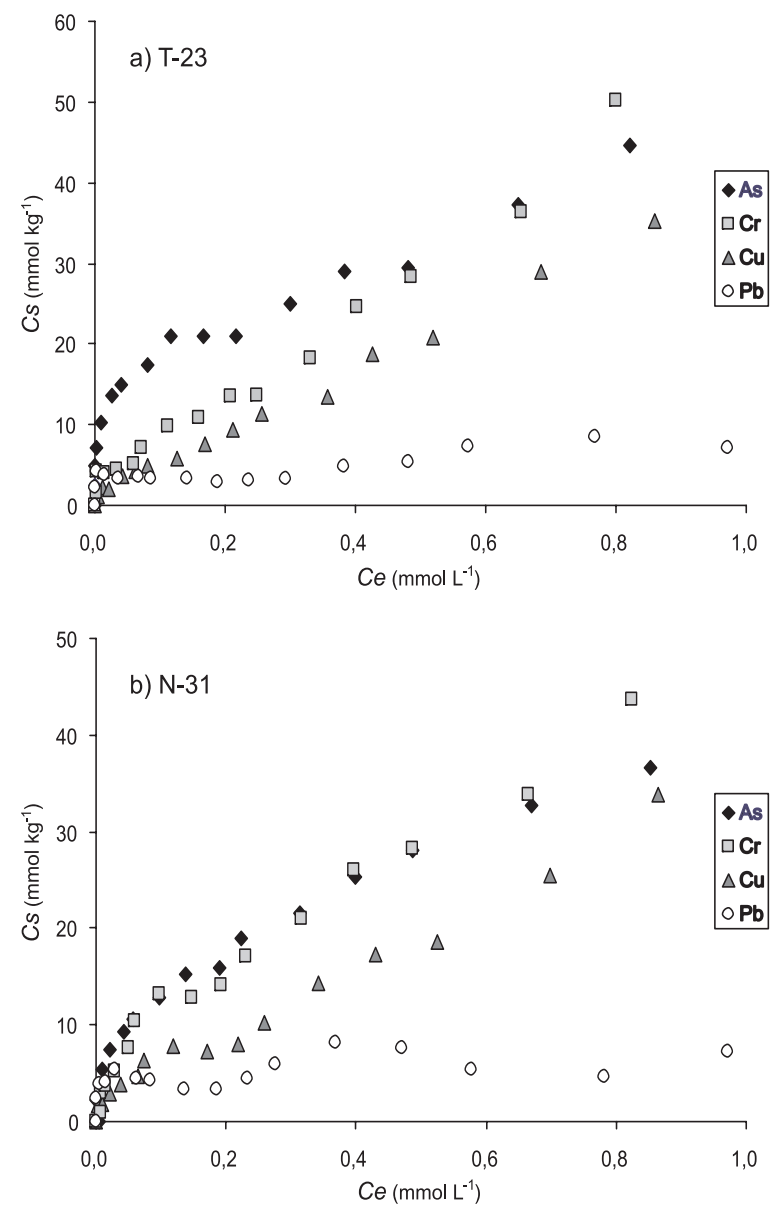

Figura 3. Isotermas competitivas para As, $\mathrm{Cu}$, Cr e Pb nos sedimentos $\mathrm{T}-23$ e $N-31$

\section{Extração sequiencial dos metais adsorvidos}

Como os valores de concentração inicial $\left(\mathrm{Co}_{a d s}\right)$ usados para cada elemento e sedimento são diferentes, o resultado da extração, apresentado na Figura 4, é dado na forma de porcentagem do metal retido em casa fase permitindo, assim, a comparação entre ambos os sedimentos. As proporções adsorvidas de cada elemento mostraram-se bastante semelhantes nos dois sedimentos quando comparados por nível competitivo. Entretanto, a distribuição dos metais nas fases extraídas após a adsorção mostra-se diferente da distribuição dos metais obtida por fracionamento dos sedimentos integrais. Nestes, exceto para o $\mathrm{Pb}$, os metais encontram-se associados quase que exclusivamente à fração menos lábil. A extração realizada após os procedimentos de adsorção mostrou que os metais se associaram preferencialmente às fases mais lábeis dos sedimentos. Esta diferença de comportamento se justifica uma vez que a extração dos sedimentos integrais mobiliza os metais adsorvidos, co-precipitados ou pertencentes à estrutura cristalina dos minerais dissolvidos em cada fração extraída. Ao se promover a adsorção, uma grande quantidade de metais será retida nos sítios superficiais disponíveis em cada fase. Estes metais adsorvidos serão totalmente mobilizados pelo processo de extração. A presença de um número maior de sítios superficiais ativos nas frações mais lábeis comparado com o número de sítios de alta energia presentes nas frações menos lábeis será responsável pela concentração do processo de adsorção nas frações trocáveis e óxido ${ }^{45}$.

Em ambos os sedimentos a adsorção não competitiva das espécies mostra que, tanto o $\mathrm{Cu}$ quanto o $\mathrm{Pb}$, são fixados preferencial- 

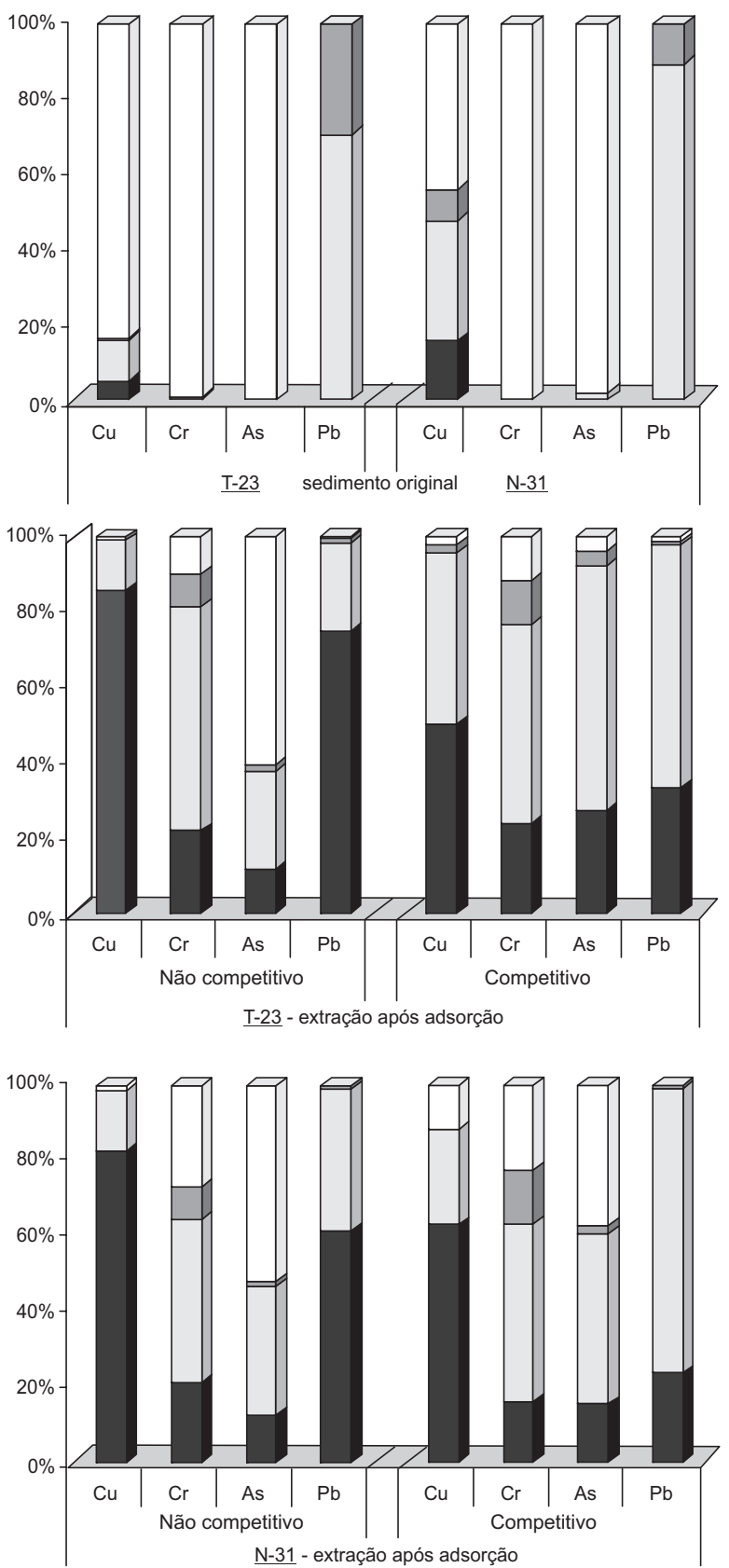

Figura 4. Resultado percentual da extração seqüencial dos sedimentos T-23 e N-31 antes e após a adsorção de $\mathrm{As}, \mathrm{Cu}, \mathrm{Cr}$ e Pb, feita para os níveis não competitivo e competitivo ( $\square$ Etapa 1, $\square$ Etapa 2, $\square$ Etapa 3, $\square$ Resíduo)

mente na fração trocáveis ( $>60 \%$ ), seguido por uma pequena porção associada à fração óxido $(\approx 15 \%)$. O Cr mostra maior afinidade com a fração óxido (60\% em T-23 e $43 \%$ em N-31) e menor quantidade associada à fração sulfeto-matéria orgânica $(<10 \%)$. Uma importante quantidade de $\mathrm{Cr}$ ocorre associada à fração residual. Esta quantidade, mais importante no sedimento N-31, representa cerca de $26 \%$ do conteúdo extraído. Diferentemente dos outros elementos, o As mostrou maior afinidade com a fração residual (> 50\% nos dois sedimentos), seguido pela fração óxido (25\% em T-23 e 35\% em N-31) e pela fração íons trocáveis ( $\approx 10 \%)$.

A partição dos elementos adsorvidos sob condições de competição se diferencia da partição observada nos sedimentos adsorvidos em condições não competitivas. No sedimento T-23, a associação do $\mathrm{Cu}$ com a fração óxido é aumentada, apresentando a seguinte distribuição: fração trocáveis $50 \%$, fração óxido $45 \%$. No sedimento N-31 observa-se a mesma tendência de aumento da participação da fração óxido na adsorção do $\mathrm{Cu}(25 \%)$. Cerca de $60 \%$ do $\mathrm{Cu}$ permanece associado à fração íons trocáveis e $10 \%$ associado à fração residual. O Cr não apresenta variação significativa da sua distribuição no sedimento T-23 sob condições competitivas. No sedimento $\mathrm{N}-31$, ocorre diminuição do $\mathrm{Cr}$ associado à fração trocáveis $(15,9 \%)$ e aumento do $\mathrm{Cr}$ associado às fases óxido $(47,5 \%)$ e sulfeto-matéria orgânica (14,3\%). O As é responsável pela maior variação entre todos os elementos. Uma importante diminuição da porcentagem de As associada à fração residual ocorre no sedimento T-23 e, em menor escala, no sedimento N-31. Essa variação indica que, sob condições competitivas, a adsorção do As ocorre preferencialmente na fração óxido. A distribuição do As passa a ser em T-23: $65 \%$ na fração óxido, $27 \%$ na fração trocáveis, $4 \%$ na fração sulfeto-matéria orgânica e 4\% na fração residual. Em N-31, $46 \%$ do As está associado à fração óxido, $37 \%$ à fração residual, $15 \%$ à fração íons trocáveis e $2 \%$ à fração sulfeto-matéria orgânica. $\mathrm{O} \mathrm{Pb}$ apresentou, sob condições competitivas, um considerável aumento da sua associação à fração óxido (64\% em T-23 e 75\% em T-31) e conseqüente diminuição de adsorção associada à fração íons trocáveis $(33,5 \%$ em T-23 e $23,6 \%$ em N-31).

\section{CONCLUSÕES}

O sedimento N-31, mais pobre em ferro, apresenta um conteúdo natural maior de metais e As associados. Entretanto, as isotermas mostram que o sedimento T-23 possui maior capacidade de adsorção para os elementos estudados. A razão para este comportamento se deve, provavelmente, à ausência de contaminantes nas águas e em torno do lago de Timbopeba. Próximos às margens do lago de Natividade, antigos garimpos de ouro podem ser a fonte dos metais e As encontrados em seu sedimento.

Em condições não competitivas, a afinidade dos elementos estudados para com os sedimentos varia de acordo com a concentração de equilíbrio. Nos dois sedimentos, para baixas concentrações de equilíbrio, a seguinte série de afinidades pode ser estabelecida: $\mathrm{Pb}>\mathrm{Cu}>\mathrm{Cr} \approx \mathrm{As}$. Para concentrações de equilíbrio mais elevadas a afinidade de adsorção se diferencia, passando a ser $\mathrm{As}>\mathrm{Pb}>\mathrm{Cu}$ $>\mathrm{Cr}$ em $\mathrm{T}-23$ e $\mathrm{As}>\mathrm{Cu}>\mathrm{Cr}>\mathrm{Pb}$ em N-31.

A comparação entre as isotermas indica que a adsorção de $\mathrm{Pb}$ diminui em condições competitivas. A competição com íons metálicos provoca um aumento na adsorção de As, provavelmente como resultado da combinação de efeitos eletrostáticos, levando ao desenvolvimento de complexos ternários superfície-metal-arsenato. As isotermas competitivas mostram que nestas condições ocorre um aumento da adsorção de $\mathrm{Cr}$ e $\mathrm{Cu}$. As causas deste comportamento não puderam ser estabelecidas neste estudo, mas podem estar relacionadas aos efeitos eletrostáticos resultantes da formação de complexos de superfície em presença do ânion arsenato.

As concentrações naturais de $\mathrm{As}, \mathrm{Cu}, \mathrm{Cr}$ estão associadas principalmente à fração residual, que é a fração menos lábil dos sedimentos, enquanto o $\mathrm{Pb}$ encontra-se associado principalmente à fração óxido. A adição de íons metálicos por adsorção aos sedimentos provocou uma mudança nesta distribuição. $\mathrm{Cu}$ e $\mathrm{Pb}$ apresentaram uma adsorção preferencial na fração trocáveis. As e Cr apresentaram maior tendência de se associarem à fração óxido. A comparação entre as quantidades de metais adsorvidas nos dois sedimentos indica maior afinidade dos metais pelo sedimento T-23. Esta afinidade pode, portanto, ser explicada pelo maior conteúdo de óxidos de ferro presente neste sedimento.

Os resultados indicam ainda que as barragens de Timbopeba e Natividade funcionam como barreiras efetivas na fixação dos ele- 
mentos estudados. A boa afinidade apresentada pelos sedimentos indica a possibilidade do seu uso na fixação de metais em ambientes contaminados. Entretanto, uma extensa proporção de metais adsorvidos foram disponibilizados na Etapa 1 do procedimento de extração seqüencial. Essa liberação evidencia que, em presença de um meio ácido complexante, boa parte dos metais adsorvidos podem ser remobilizados para o meio aquático.

\section{AGRADECIMENTOS}

Ao CNPq (projeto ${ }^{\circ}$ 910018), ao BMBF da Alemanha (WTZ Proj. ${ }^{\circ}$ BRA 99/043 ENV) e à CAPES (projeto $n^{\circ} 162 / 03$ ), ao DAAD (Proj. $n^{\circ}$ 415-br-probral/ale-02/21604), responsáveis pelo financiamento deste estudo. À Cia. Vale do Rio Doce (Mina de Timbopeba) pelo suporte nos trabalhos de campo. Ao Prof. Dr. H. Pöllmann da Martin-Luther-University of Halle-Wittenberg e ao UFZ - Centre for Environmental Research Leipzig-Halle, Magdeburg.

\section{REFERÊNCIAS}

1. Benjamin, M. M.; Lekie, J. O.; J. Colloid Interface Sci. 1981, 79, 209.

2. Tessier, A.; Rapin, F.; Carignan, R.; Geochim. Cosmochim. Acta 1985, 49, 183.

3. Cornell, R. M.; Schwertmann, U.; The Iron Oxides, Wiley-VCH: Weinheim, 1996.

4. Garcia-Sánchez, A.; Alastuey, A.; Querol, X.; Sci. Total Environ. 1999, 242, 179.

5. Tessier, A.; Campbell, P. G. C.; Bisson, M.; Anal. Chem. 1979, 51, 844.

6. Salomons, W.; Förstner, U.; Environ. Technol. Lett. 1980, 1, 506.

7. Bogle, E. W; Nichol, I.; J. Geochim. Explor. 1981, 15, 405.

8. Gleyzes, C.; Tellier, S.; Astruc, M.; Trends Anal. Chem. 2002, 21, 451.

9. Hlavay, J.; Prohaska, T.; Weisz, M; Wenzel, W. W.; Stingeder, G.; J. Pure Appl. Chem. 2004, 76, 415.

10. Quevauviller, Ph.; Rauret, G.; López-Sànchez, J. F.; Rubio, R.; Ure, A. M.; Muntau, H.; Sci. Total Environ. 1997, 205, 223.

11. Ure, A. M.; Davidson, C. M.; Chemical Speciation in the Environment, $2^{\text {nd }}$ ed., Blackwell Scientific Publications: Oxford, 2002.

12. Quevauviller, Ph. Em Methodologies for Soil and Sediment Fractionation Studies; Quevauviller, Ph., ed.; RSC: Cambridge, 2002, cap 1.

13. Costa, A. C. S.; Almeida V. C.; Lenzi E.; Nozaki J.; Quim. Nova 2002, 25 , 548.

14. Bacon, J. R.; Hewitt, I. J.; Cooper, P.; Sci. Total Environ. 2005, 337, 191.

15. Sodre, F. F.; Lenzi, E.; Costa, A. C. S.; Quim. Nova 2001, 24, 324.

16. Jordão, C. P.; Alves, N. M.; Pereira J. L.; Bellato, C. R.; Alvarez, V. H.; Quim. Nova 2000, 23, 5.

17. Smith, K. S. Em The Environmental Geochemistry of Mineral Deposits, Part A; Plumlee, G. S.; Logsdon, M. J., eds.; Society of Economic Geologists, Reviews in Economic Geology, 1999, cap 7.
18. Stumm, W.; Chemistry of the Solid-Water Interface, Wiley-Interscience: New York, 1992.

19. Davis, J. A.; Leckie, J. O.; J. Colloid Interface Sci. 1980, 67, 90.

20. Dzombak, D. A.; Morel, F. M. M.; Surface Complexation Modeling hydrous ferric oxide, John Wiley and Sons: New York, 1990.

21. Eleutério, L.; Dissertação de Mestrado, Universidade Federal de Ouro Preto, Brasil, 1997.

22. Costa, A. T.; Dissertação de Mestrado, Universidade Federal de Ouro Preto, Brasil, 2001.

23. Matschullat, J.; Borba, R. P.; Deschamps, E.; Figueiredo, B. R.; Gabrio, T.; Schwen, M.; Appl. Geochem. 2000, 15, 181.

24. Basílio, M. S.; Nalini Jr., H. A.; Friese, K.; Roeser, H. M. P.; Resumos Expandidos do IX Congresso Brasileiro de Geoquímica, Belém do Pará, Brasil, 2003.

25. Müller, I.; Pluquet, E.; Water Sci. Technol. 1998, 37, 379.

26. Ding, M.; De Jong, B. H. W. S.; Roosendaal, S. J.; Vredenberg, A.; Geochim. Cosmochim. Acta 2000, 64, 1209.

27. Norman, D. I.; Ndur, S.; Appiah, H.; Nartey, R. S.; Nkrumah, K.; 5th International Arsenic Exposure and Health Effects Conference - Poster Abstracts, San Diego, Estados Unidos, 2002.

28. Bose, P.; Bose, M. A.; Kumar, S.; Adv. Environ. Res. 2002, 7, 179.

29. Zhang, Y.; Yang, M.; Huang, X.; Chemosphere 2003, 51, 945.

30. Aguiar, M. R. M. P.; Novaes. A. C.; Guarino, A. W. S.; Quim. Nova 2002, $25,1145$.

31. Guedes, C. D.; Pereira, J. G.; Lena, J. C.; Paiva, J. F.; Lima, R. M. F.; Quim. Nova 2004, 27, 715 .

32. Echeverría, J. C.; Morera, M. T.; Mazkiarán, C.; Garrido, J. J.; Environ. Pollut. 1998, 101, 275.

33. Echeverría, J. C.; Morera, M. T.; Garrido, C.; Aust. J. Soil Res. 1999, 37, 431.

34. Dong, D.; Nelson, Y. M.; Lion, L. W.; Shuler, M. L.; Ghiorse, W. C.; Water Res. 1999, 34, 427.

35. Veeresh, H.; Tripathy, S.; Chaudhuri, D.; Hart, B. R.; Powell, M. A.; Appl. Geochem. 2003, 18, 1723.

36. Ure, A.; Queveuviller, Ph.; Muntau, H.; Griepink, B.; Int. J. Environ. Anal. Chem. 1993, 51, 135.

37. Rauret, G.; López-Sánches, J. F.; Lück, D.; Yli-Halla, M.; Muntau, H.; Quevauviller, Ph.; The certification of the extractable contents (mass fractions) of $\mathrm{Cd}, \mathrm{Cr}, \mathrm{Cu}, \mathrm{Ni}, \mathrm{Pb}$ and $\mathrm{Zn}$ in freshwater sediment following sequential extraction procedure - BCR 701, BCR information reference material: Bruxelas, Bélgica, 2001.

38. Davidson, C. M.; Duncan, A. L.; Littlejohn, D.; Ure, A. M.; Garden, L. M.; Anal. Chim. Acta 1998, 363, 45.

39. Ryan, P. C.; Wall, A. J.; Hillier, S.; Clark, L.; Chem. Geol. 2002, 184, 337.

40. Tokaliolu, Ş.; Kartal, Ş.; Elçi, L.; Anal. Chim. Acta 2000, 413, 33.

41. Gómez-Ariza, J. L.; Giráldez, I.; Sánchez-Rodas, D.; Morales, E.; Anal. Chim. Acta 1999, 399, 295.

42. Brunauer, S.; Deming, L.; Deming, W.; Teller, E.; J. Am. Chem. Soc. 1940 , 62, 1723.

43. Khaodhiar, S.; Azizian, M. F.; Osathaphan, K.; Nelson, P. O.; Water, Air, Soil Pollut. 2000, 119, 105.

44. Christl, I.; Kretzschmar, R.; Geochim. Cosmochim. Acta 1999, 63, 2929.

45. Morera, M. T.; Echeverría, J. C.; Mazkiarán, C.; Garrido, J. J.; Environ. Pollut. 2001, 113, 135. 